\title{
Incomplete Oophorectomy
}

National Cancer Institute

\section{Source}

National Cancer Institute. Incomplete Oophorectomy. NCI Thesaurus. Code C29893.

Surgery that does not remove the entire ovary. This can be by design or by error. 